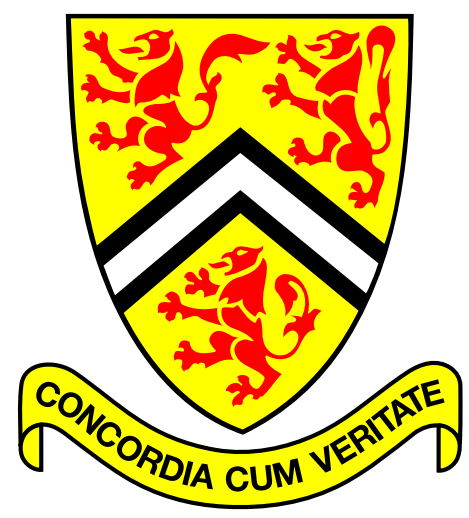

\title{
PAPR Reduction Using Integer Structures in OFDM Systems
}

\author{
Amin Mobasher and Amir K. Khandani \\ Coding \& Signal Transmission Laboratory \\ Department of Electrical \& Computer Engineering \\ University of Waterloo \\ Waterloo, Ontario, Canada, N2L 3G1 \\ Technical Report UW-E\&CE\#2004-06 \\ April 30, 2004
}




\title{
PAPR Reduction Using Integer Structures in OFDM Systems
}

\author{
Amin Mobasher and Amir K. Khandani \\ Coding \& Signal Transmission Laboratory (www.cst.uwaterloo.ca) \\ Dept. of Elec. and Comp. Eng., University of Waterloo \\ Waterloo, Ontario, Canada, N2L 3G1 \\ Tel: 519-884-8552, Fax: 519-888-4338 \\ e-mail: \{amin, khandani\}@ cst.uwaterloo.ca
}

\begin{abstract}
In this work, the problem of reducing the Peak to Average Power Ratio (PAPR) in an Orthogonal Frequency Division Multiplexing (OFDM) system is considered. We design a cubic constellation, called the Hadamard constellation, whose boundary is along the bases defined by the Hadamard matrix in the transform domain. Then, we further reduce the PAPR by applying the Selective Mapping technique. The encoding algorithm is derived from a decomposition, known as the Smith Normal Form (SNF), and has a minimal complexity. This new technique offers a PAPR that is approximately $2 \mathrm{~dB}$ to $3 \mathrm{~dB}$ lower than that of the best known techniques without any lose in terms of energy and/or spectral efficiency and without any side information being transmitted. Moreover, it has a low computational complexity.
\end{abstract}




\section{INTRODUCTION}

Orthogonal Frequency Division Multiplexing (OFDM) is a multicarrier transmission technique which is widely adopted in different communication applications. OFDM prevents Inter Symbol Interference (ISI) by inserting a guard interval and mitigates the frequency selectivity of a multi-path channel by using a simple equalizer. This simplifi es the design of the receiver and leads to inexpensive hardware implementations. Moreover, OFDM offers some advantages in higher order modulations and in the networking operation that position OFDM as the technique of choice for the next generation of wireless networks. However, OFDM systems have the undesirable feature of a large Peak to Average Power Ratio (PAPR) of the transmitted signals. Consequently to prevent the spectral growth of the OFDM signal, the transmit amplifi er must operate in its linear regions. Therefore, power amplifi ers with a large linear range are required for OFDM systems, but such amplifi ers will continue to be a major cost component of OFDM systems. Consequently, reducing the PAPR is pivotal to reducing the expense of OFDM systems.

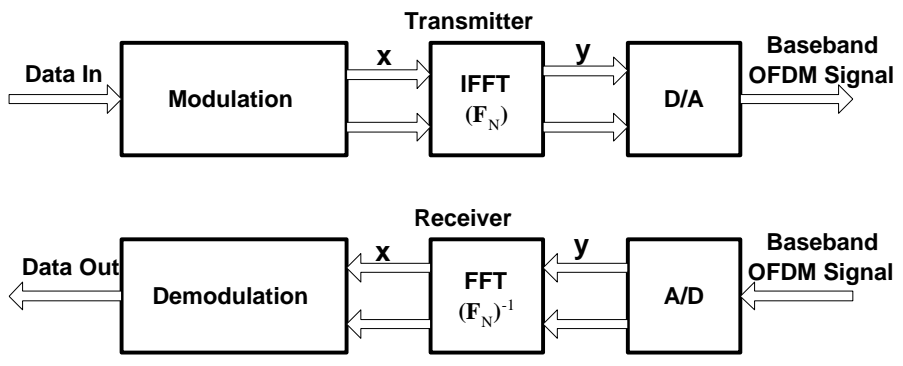

Fig. 1. Basic OFDM transmitter and receiver.

Fig. 1 shows a basic block diagram of an OFDM transmitter and receiver. Let $\mathbf{x}=\left\{x_{0}, x_{1}, \cdots, x_{N-1}\right\}$ denote a vector of $2 N$ Dimensional (2N-D) constellation point selected from a set of $N$ identical 2-D sub-constellations, $\left\{x_{0}, x_{1}, \cdots, x_{N-1}\right\}$, to be transmitted by using one OFDM vector of size $N$; namely, $\mathbf{y}$. 
The discrete time samples of the OFDM signal can be expressed as

$$
y_{n}=\frac{1}{\sqrt{N}} \sum_{k=0}^{N-1} x_{k} e^{j 2 \pi \frac{n k}{N}} .
$$

The matrix representation of this signal is

$$
\mathbf{y}=\mathbf{F}_{N} \mathbf{x}
$$

where $\mathbf{y}=\left(y_{0} \cdots y_{N-1}\right)^{T}, \mathbf{x}=\left(x_{0} \cdots x_{N-1}\right)^{T}$, and $\mathbf{F}_{N}$ is the IFFT matrix,

$$
\mathbf{F}_{N}=\frac{1}{\sqrt{N}}\left[\begin{array}{ccccc}
1 & \cdots & 1 & \cdots & 1 \\
\vdots & \ddots & \vdots & \ddots & \vdots \\
1 & \cdots & e^{j 2 \pi \frac{n k}{N}} & \cdots & e^{j 2 \pi \frac{n(N-1)}{N}} \\
\vdots & \ddots & \vdots & \ddots & \vdots \\
1 & \cdots & e^{j 2 \pi \frac{k(N-1)}{N}} & \cdots & e^{j 2 \pi \frac{(N-1)^{2}}{N}}
\end{array}\right]
$$

The 2-D constellation points, $\left\{x_{0}, x_{1}, \cdots, x_{N-1}\right\}$, may add constructively and produce a time domain signal with a large amplitude. Thus, the output signal y can have high output levels, which leads to the requirement of an expensive analog front end.

Usually, the level of the amplitude fluctuation of the discrete time OFDM signal is measured in terms of the peak factors that indicate the ratio of the peak power to the average envelop power of the signal as

$$
\operatorname{PAPR}(\mathbf{y})=\frac{\max _{i}\left|y_{i}\right|^{2}}{E_{y}\left[\frac{1}{N}\|\mathbf{y}\|^{2}\right]} .
$$

Also, the continuous time PAPR is typically estimated by the discrete time PAPR by employing the IFFT of length $L N$ for the zero padded sequence of length $L N$ derived from the sequence $\left\{x_{0}, x_{1}, \cdots, x_{N-1}\right\}$ in (1) [1-3]. Therefore,

$$
y_{n}=y(n T)=\frac{\sqrt{L}}{\sqrt{L N}} \sum_{k=0}^{L N-1} x_{k}^{\prime} e^{j 2 \pi \frac{n k}{L N}}
$$


where

$$
x_{k}^{\prime}= \begin{cases}x_{k}, & \text { for } k<N \\ 0, & \text { for } k \geq N .\end{cases}
$$

and $L$ is the oversampling factor.

Lastly, (7) is the real representation of the complex equation in (2).

$$
\left[\begin{array}{c}
\operatorname{Re}(\mathbf{y}) \\
\operatorname{Im}(\mathbf{y})
\end{array}\right]=\left[\begin{array}{cc}
\operatorname{Re}\left(\mathbf{F}_{N}\right) & -\operatorname{Im}\left(\mathbf{F}_{N}\right) \\
\operatorname{Im}\left(\mathbf{F}_{N}\right) & \operatorname{Re}\left(\mathbf{F}_{N}\right)
\end{array}\right]\left[\begin{array}{c}
\operatorname{Re}(\mathbf{x}) \\
\operatorname{Im}(\mathbf{x})
\end{array}\right] .
$$

Since we focus on only real matrices, we use the same notation as (2) for (7).

A large number of methods for the PAPR reduction have been proposed [3-18]. In $[4,5]$, coding techniques are used for PAPR reduction; however, codes offering a low PAPR can be constructed only at the cost of sacrifi cing the data rate.

Clipping the OFDM signal before amplifi cation is a simple and typical method for the PAPR reduction [6-8]. In $[3,9,10]$, the effect of oversampled and clipped OFDM signals are analyzed.

There are two probabilistic schemes to reduce the PAPR. One is the Partial Transmit Sequence (PTS) [11] in which each block of subcarriers is multiplied by a constant phase factor, and these phase factors are optimized to minimize the PAPR. The other scheme is Selective Mapping (SLM) in which multiple sequences are generated from the same information, and the sequence with the lowest PAPR is transmitted [12-14]. Typically, the receiver needs to know which sequence is selected in order to recover the data. However, the methods introduced in [11-14] eliminate the need for this explicit side information.

In [15], a new constellation technique is developed. It extends the outer constellation points to minimize the PAPR of the OFDM symbol. In [16-18], a constellation shaping technique is proposed to reduce the PAPR of the OFDM signals. The encoding and decoding algorithms of this method are based on the relations and generators in a free Abelian group. Due to the large complexity of this algorithm, its practical implementation is very challenging.

In this paper, we proposed a constellation as a shaping method in an OFDM system with a low complexity encoding method, based on [16], and a considerable PAPR reduc- 
tion. An SLM method is applied in conjunction with our constellation to further reduce the PAPR in the OFDM signals.

The rest of this paper is organized as follows. In Section II, constellation shaping is introduced. A brief description of the work in [16] is also given. Section III describes the Hadamard constellation as a shaping method in OFDM systems. Some issues of the encoding and decoding algorithms are also investigated. An SLM method is applied to the Hadamard constellation in Section IV. Section V is devoted to some numerical results and a comparison of our method with some recent works. The paper is concluded in Section VI.

\section{Constellation Shaping}

In constellation shaping, a constellation in the frequency domain must be found such that the resulting shaping region in the time domain has a low PAPR. A new constellation shaping method is introduced in [16-18] by Kwok and Jones. Based on the encoding algorithm introduced in [16], we propose a cubic constellation, along with an SLM method to reduce the PAPR in an OFDM system.

In a PAPR reduction problem, the peak value of the signal vector should be bounded by a specifi ed value $\|\mathbf{y}\|_{\infty} \leq \beta$. If the real time signal is related to the real frequency constellation point by $\mathbf{y}=\mathbf{A x}$, this inequality on the time domain boundary translates to a parallelotope $^{1}$ in the frequency domain, defi ned by $\mathbf{A}^{-1}$. The points inside this parallelotope are used as constellation points in transmitting the OFDM signals. The principal challenge in constellation shaping is to find a unique way to map the input data to the constellation points such that the mapping (encoding) and its inverse (decoding) can be implemented by a reasonable complexity. In [16], the relations and generators in a free Abelian group in the integer domain are used so that the parallelotope corners lie in the integer lattice. Therefore, the constellation boundary is based on a parallelotope, defi ned by $\mathbf{Q}_{N}=\left[\alpha \mathbf{A}^{-1}\right]$, where [.] represents rounding, and $\alpha$ is the smallest value to have the same constellation points as those of the unshaped constellation.

\footnotetext{
${ }^{1}$ The parallelotope bases are defi ned along the columns of $\mathbf{A}^{-1}$.
} 
The shaped constellation for an OFDM system is the quotient group, $\mathcal{Z}^{N} / \Lambda\left(\mathbf{Q}_{N}\right)$, where $\mathcal{Z}^{N}$ is the $N$-D integer space and $\Lambda\left(\mathbf{Q}_{N}\right)$ is the lattice defi ned by $\mathbf{Q}_{N}$. The encoding of this constellation is performed by the decomposition of the relation matrix $\mathbf{Q}_{N}$, based on column and row operations [16]. Indeed, in the mathematical literature this decomposition is known as the Smith Normal Form (SNF) of an integer matrix [19].

The SNF is a diagonalization of a matrix in the integer domain. Introduced by Smith [20], this concept has been used in many applications in many fi elds such as solving linear diophantine equations, fi nding the permutation equivalence and similarity of matrices, determining the canonical decomposition of the fi nitely generated Abelian groups, integer programming, computing additional normal forms, including Frobenius and Jordan normal forms, and separable computing of the discrete Fourier transform. For more historical remarks and applications of the SNF, see [21-23].

The SNF is well known theoretically, but can be diffi cult to compute in practice because of the potential for rapid growth in the size of the intermediate expressions. However, there are a number of algorithms to compute the SNF decomposition of an integer matrix, and there are some polynomial time algorithms for this decomposition in special cases [22, 24-27]. The following theorem and its corresponding algorithms can be used, instead of the column and row operations in [16].

Theorem 1: Any integer matrix $\mathbf{Q}_{N}$ can be decomposed into $\mathbf{Q}_{N}=\mathbf{U D V}$, where $\mathbf{D}$ is diagonal with the entries $\left\{\sigma_{i}\right\}_{i=1}^{N}$ such that $\sigma_{1}\left|\sigma_{2}\right| \cdots \mid \sigma_{N}$, and $\mathbf{U}$ and $\mathbf{V}$ are unimodular matrices. The matrix $\mathbf{D}$ is called the $\mathrm{SNF}$ of the matrix $\mathbf{Q}_{N}$ [19]. The condition $\sigma_{1}\left|\sigma_{2}\right| \cdots \mid \sigma_{N}$ in Theorem 1 is defi ned for fi nding a unique decomposition and can be ignored in the encoding procedure.

The complexity of this algorithm is the result of the computation of the SNF decomposition for the matrix $\mathbf{Q}_{N}$ which is based on rounding off the scaled version of the IFFT matrix. We can use the SNF decomposition methods for the encoding procedure; however, the computational complexity for OFDM systems that are defi ned by the IFFT matrix remains very high. 
In [17], it is shown that if the matrix $\mathbf{Q}_{\mathbf{N}}$ is replaced by the Hadamard matrix, the encoding and decoding algorithm for the constellation based on this matrix can be implemented by a butterfly structure that uses only bit shifting and logical AND. This simplicity is hidden in the following recursive formula for the Hadamard matrix:

$$
\mathbf{H}_{2^{n}}=\left[\begin{array}{cc}
\mathbf{H}_{2^{n-1}} & \mathbf{H}_{2^{n-1}} \\
\mathbf{H}_{2^{n-1}} & -\mathbf{H}_{2^{n-1}}
\end{array}\right], \text { where } \mathbf{H}_{1}=[1] \text {. }
$$

The SNF of (8) can be easily computed in (9).

$$
\begin{gathered}
\mathbf{U}_{2^{n}}=\left[\begin{array}{cc}
\mathbf{U}_{2^{n-1}} & 0 \\
\mathbf{U}_{2^{n-1}} & \mathbf{U}_{2^{n-1}}
\end{array}\right] \mathbf{D}_{2^{n}}=\left[\begin{array}{cc}
\mathbf{D}_{2^{n-1}} & 0 \\
0 & 2 \mathbf{D}_{2^{n-1}}
\end{array}\right] \\
\mathbf{V}_{2^{n}}=\left[\begin{array}{cc}
\mathbf{V}_{2^{n-1}} & \mathbf{V}_{2^{n-1}} \\
0 & -\mathbf{V}_{2^{n-1}}
\end{array}\right] \mathbf{U}_{2^{n}}^{-1}=\left[\begin{array}{cc}
\mathbf{U}_{2^{n-1}} & 0 \\
-\mathbf{U}_{2^{n-1}} & \mathbf{U}_{2^{n-1}}
\end{array}\right],
\end{gathered}
$$

where $\mathbf{U}_{1}=\mathbf{U}_{1}^{-1}=\mathbf{D}_{1}=\mathbf{V}_{1}=[1]$.

Therefore, the encoding algorithm for this constellation can be represented by [16]

$$
\begin{aligned}
& \hat{\mathbf{x}}=\mathbf{U}_{N} \boldsymbol{\lambda} \\
& \boldsymbol{\gamma}=\left\lfloor\frac{\mathbf{H}_{N}^{T} \hat{\mathbf{x}}}{N}\right\rfloor \\
& \mathbf{x}=\hat{\mathbf{x}}-\mathbf{H}_{N} \boldsymbol{\gamma} \\
& \mathbf{y}=\operatorname{IFFT}(\mathbf{x}),
\end{aligned}
$$

where $N=2^{n}$, and $\boldsymbol{\lambda}$ is the canonical representation of integers $I$ representing the constellation points. The canonical representation of any integer can be calculated by the recursive modulo operations; namely,

$$
\begin{aligned}
\lambda_{1} & =I \bmod \sigma_{1} \\
I_{1} & =\frac{I-\lambda_{1}}{\sigma_{1}} \\
\lambda_{i} & =I_{i-1} \bmod \sigma_{i} \\
I_{i} & =\frac{I_{i-1}-\lambda_{i}}{\sigma_{i}},
\end{aligned}
$$


where $1 \leq i \leq N$.

The reverse operation for fi nding $I$ from the $N$-D vector $\mathbf{x}$ is

$$
\begin{aligned}
& \boldsymbol{\lambda}=\mathbf{U}_{N}^{-1} \mathbf{x}=\left(\lambda_{1}, \lambda_{2}, \cdots, \lambda_{N}\right)^{T}, \\
& \tilde{\lambda}_{i}=\lambda_{i} \bmod \sigma_{i}, \\
& I=\tilde{\lambda}_{1}+\sigma_{1}\left(\tilde{\lambda}_{2}+\sigma_{2}\left(\cdots\left(\tilde{\lambda}_{N-1}+\sigma_{N-1} \tilde{\lambda}_{N}\right) \cdots\right)\right) .
\end{aligned}
$$

\section{HADAMARD CONSTELLATION IN OFDM SySTEMS}

As mentioned in Section II, if the IFFT operations in OFDM multicarrier modulation could be changed by the Hadamard matrix, a very simple encoding algorithm would result. However, this type of multicarrier modulation is not very popular because it does not offer all the advantages of conventional OFDM systems [28]. The constellation that should be used in an OFDM system has a boundary along the bases of the IFFT matrix, but the encoding of containing constellation points cannot be easily implemented. We propose a cubic constellation, called the Hadamard constellation, for an OFDM system whose boundary is along the bases defi ned by the Hadamard matrix in the transform domain. The IFFT and Hadamard matrices are both orthogonal matrices, and therefore, the constellations along these orthogonal bases are a rotated version of each other. This idea is illustrated in Fig. 2. By substituting the proper constellation along the IFFT matrix by the Hadamard matrix in an OFDM system, the resulting PAPR is reduced; however, the encoding of this constellation, based on the SNF decomposition of the Hadamard matrix, is simple and practical. Moreover, the encoding algorithm can be implemented by a butterfly structure that uses bit shifting and logical AND structures [16].

The advantage of using the Hadamard constellation is not only a simple encoding algorithm with a low PAPR, but also the Hadamard constellation's ability to be concatenated with other methods. This motivates us to apply a Selective Mapping (SLM) technique $[29,30]$ to the Hadamard constellation in an OFDM system. In typical SLM methods $[29,30]$, the major PAPR reduction is achieved by the fi rst few redundant bits. Employing more redundant bits necessitates a high level of complexity to obtain modest improvements 
in the PAPR value. However, in the proposed SLM method, employing the Hadamard constellation causes a considerable PAPR reduction by itself. As a result, this method, by just using one or two redundant bits in SLM, signifi cantly outperforms the other PAPR reduction techniques, reported in the literature. The details of this method will be explained in the next section and will be confi rmed by simulation results. In the following, we have investigated some issues that have emerged regarding the use of the Hadamard constellation in an OFDM system.

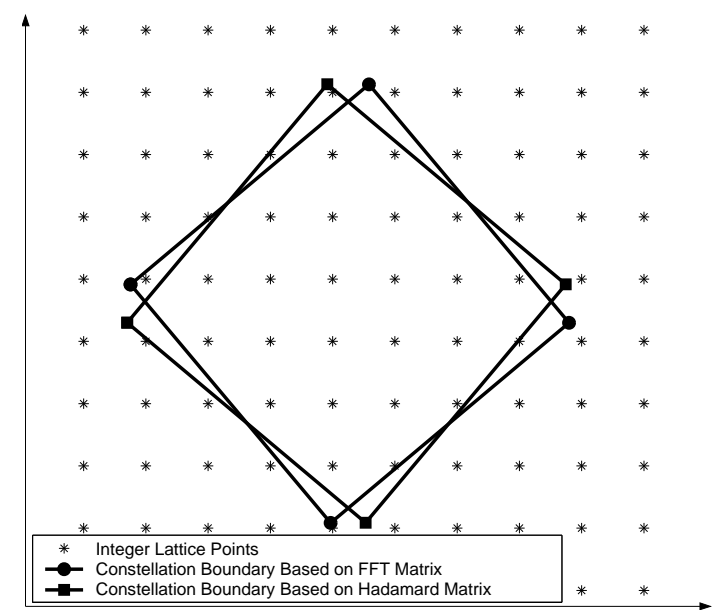

Fig. 2. $N$-D signal constellation for IFFT and Hadamard matrix.

\section{A. Complex Approximation}

As stated in Section II, (7) can be applied to change the complex equations of an OFDM system to real equations. This leads to the change of the constellation boundary. Generally, we can distinguish between two classes of boundaries [31, 32]: 1) Cartesian boundary that is resulted by viewing the real and imaginary parts of the signal as two separate real signals, and 2) polar boundary that considers the envelope and phase of the OFDM signal in a complex plain. Cartesian boundary limits each component of the complex signal within a square, while the polar boundary limits this component within a circle. In this paper, we avoid the complex representation of the OFDM signal by treating the real 
and imaginary parts of the signal independently, which is equivalent to using a Cartesian boundary.

\section{B. Encoding Procedure}

All the points inside the Hadamard constellation should be labeled by the encoding procedure, introduced in (10) - (12). The number of points, inside the shaped constellation is determined by the determinant of the Hadamard matrix, $\operatorname{det}\left(\mathbf{H}_{2^{n}}\right)$.

Theorem 2: The constellation size for a $2^{n} \times 2^{n}$ Hadamard matrix is $\operatorname{det}\left(\mathbf{H}_{2^{n}}\right)=$ $2^{n 2^{n-1}}$.

Proof: Based on (9), $\operatorname{det}\left(\mathbf{H}_{2^{n}}\right)=\operatorname{det}\left(\mathbf{D}_{2^{n}}\right)$, because the matrices $\mathbf{U}_{\mathbf{2}^{\mathbf{n}}}$ and $\mathbf{V}_{\mathbf{2}^{\mathbf{n}}}$ are unimodular and their determinant is one. To prove this theorem, we use deduction. For a $2 \times 2$ Hadamard matrix,

$$
\operatorname{det}\left(\mathbf{D}_{2}\right)=\operatorname{det}\left(\left[\begin{array}{ll}
1 & 0 \\
0 & 2
\end{array}\right]\right)=2=2^{1 \times 2^{1-1}} .
$$

It is assumed that the claim is valid for a $2^{k} \times 2^{k}$ Hadamard matrix. Based on (9), for a $2^{k+1} \times 2^{k+1}$ Hadamard matrix,

$$
\begin{gathered}
\mathbf{D}_{2^{k+1}}=\left[\begin{array}{cc}
\mathbf{D}_{2^{k}} & 0 \\
0 & 2 \mathbf{D}_{2^{k}}
\end{array}\right] \\
\Rightarrow \operatorname{det}\left(\mathbf{D}_{2^{k+1}}\right)=\operatorname{det}\left(\mathbf{D}_{2^{k}}\right) \times 2^{2^{k}} \times \operatorname{det}\left(\mathbf{D}_{2^{k}}\right) \\
\Rightarrow \operatorname{det}\left(\mathbf{D}_{2^{k+1}}\right)=2^{2^{k}} \times\left(\operatorname{det}\left(\mathbf{D}_{2^{k}}\right)\right)^{2} \\
\Rightarrow \operatorname{det}\left(\mathbf{D}_{2^{k+1}}\right)=2^{2^{k}} \times\left(2^{k 2^{k-1}}\right)^{2}=2^{(k+1) 2^{(k+1)-1}} .
\end{gathered}
$$

According to the large Hadamard constellation size, the canonical representation of the large numbers should be computed in the transmission of the OFDM signals. The canonical representation can be simplifi ed by using the fact that digital communication 
systems deal with binary input streams. Based on (12) in the encoding procedure, any Integer $I$ can be represented by

$$
I=\lambda_{1}+\sigma_{1} \lambda_{2}+\sigma_{1} \sigma_{2} \lambda_{3}+\cdots+\sigma_{1} \ldots \sigma_{N-1} \lambda_{N}
$$

where $N=2^{n}$, and $\left\{\lambda_{i}\right\}_{i=1}^{N}$ is the canonical representation of $I$ with $\lambda_{1}=0$. Based on (9), for a $2^{n} \times 2^{n}$ Hadamard matrix, all $\left\{\sigma_{i}\right\}_{i=1}^{2^{n}}$ are powers of 2 such that

$$
\left\{\sigma_{i}\right\}_{i=1}^{2^{n}}=\left\{1,2,2,4,2,4,4,8, \cdots, 2^{n}\right\}
$$

Therefore,

$$
I=\lambda_{2}+2 \lambda_{3}+2^{2} \lambda_{4}+2^{4} \lambda_{5} \cdots+2^{n 2^{n-1}-n} \lambda_{N}
$$

The representation of $d=2^{n 2^{n-1}}$ integers necessitate that $n_{b}=\log _{2}(d)=n 2^{n-1}$ bits, and thus, the binary representation of $I$ is expressed as

$$
\begin{aligned}
I= & b_{0}+2 b_{1}+2^{2} b_{2}+2^{3} b_{3}+\cdots+2^{n_{b}-1} b_{n_{b}-1} \\
= & b_{0}+2 b_{1}+2^{2}\left(b_{2}+2 b_{3}\right)+2^{4} b_{4}+2^{5}\left(b_{5}+b_{6}\right)+ \\
& \cdots+2^{n 2^{n-1}-n}\left(b_{n_{b}-n}+\cdots+2^{n-1} b_{n_{b}-1}\right)
\end{aligned}
$$

A comparison of (17) and (18) is depicted in Fig. 3. This representation will simplify the encoding algorithm. Moreover, the problem of using large numbers in the encoding procedure will be avoided.

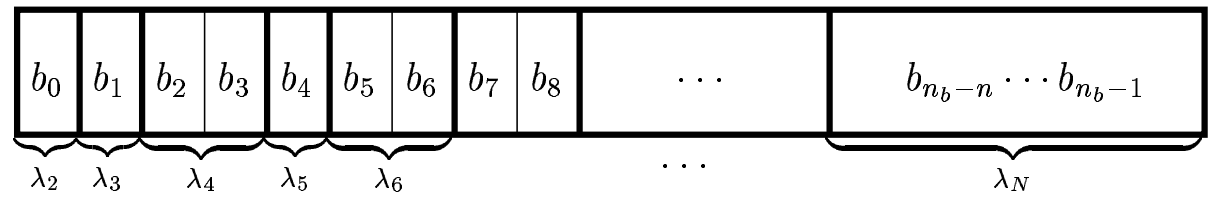

Fig. 3. Mapping between binary representation of the information and $\left\{\lambda_{i}\right\}$.

Theorem 2 shows the size of the Hadamard constellation for a $2^{n} \times 2^{n}$ Hadamard matrix is $2^{n 2^{n-1}}$. Therefore, the transmission rate is related to the number of subcarriers $N=2^{n}$ in the OFDM system ${ }^{2}$. This rate is unacceptable not only because of the dependency on $N$ but also because the value is usually much higher than the required rate. A

$$
{ }^{2} \text { For } N=2^{n} \text {, the rate for each real component is } \log _{2}\left(2^{n 2^{n-1}}\right) / N=\frac{n}{2} \text {. }
$$


selection method for the constellation points must be implemented such that the constellation has the desired rate, and the constellation points have a uniform distribution in the cubic constellation.

Noting (10) and (11), there is an isomorphism between the integer set

$$
\mathcal{S}=\left\{0,1, \cdots, 2^{n 2^{n-1}}-1\right\}
$$

and the set of the points within the Hadamard constellation. Equivalently, the set $\mathcal{S}$ can be considered as a label group for the constellation points (refer to [33] for the defi nition). A subgroup of constellation points results in a uniformly distributed subset of the Hadamard constellation points. Consequently, this subgroup of constellation points is isomorphic to a subgroup in the label group $\mathcal{S}$. This subgroup can be selected such that its elements are congruent to zero modulo $c$, namely

$$
\mathcal{P}=\{I \in \mathcal{S} \mid I \equiv 0(\bmod c)\}
$$

where $c$ is determined by the ratio of the size of the Hadamard constellation, $2^{n 2^{n-1}}$, and the size of the constellation, $2^{r N}$, with the desired rate, $r$. Employing (10) and (11), the labels in the subgroup $\mathcal{P}$ determine the set of uniformly distributed points in the Hadamard constellation. Relying on the continuous approximation, such a uniform distribution affects neither the probabilistic behavior of the PAPR nor the average energy of the constellation points. Note that the Hadamard constellation is called the root constellation for the aforementioned set of the uniformly distributed points in the sequel.

\section{Decoding Procedure}

A conventional Fast Fourier Transform (FFT) based receiver is considered for the OFDM signal. At the receiver end, the time domain signal is filtered by a low pass fi lter and sampled at the Nyquist rate. The samples are processed by an FFT to recover the constellation point in the frequency domain. For an Additive White Gaussian Noise (AWGN) channel, the received vector is given by

$$
\mathbf{z}=\mathbf{y}+\mathbf{n}
$$


where $\mathbf{y}$ is the transmitted time domain signal in (10) and $\mathbf{n}$ is a zero-mean complex AWGN. The approximated constellation point is written as

$$
\hat{\mathbf{x}}=\operatorname{FFT}(\mathbf{z})=\mathbf{x}+\operatorname{FFT}(\mathbf{n})=\mathbf{x}+\mathbf{n}^{\prime},
$$

where $\mathbf{x}$ is the transmitted constellation point, and $\mathbf{n}^{\prime}$ is a zero-mean complex AWGN. The maximum likelihood decoder simply rounds off the received constellation point $\hat{\mathbf{x}}$ in the integer domain. Then, the resulting constellation point is employed in (12) to decode the transmitted information.

\section{Selective MapPing}

As mentioned in Section III, the complexity of using the Hadamard constellation in an OFDM system is very low, and this shaping method can be concatenated by other methods. In the following, we propose an SLM technique, applied to the Hadamard constellation to further reduce the PAPR.

SLM is a method to reduce the PAPR in an OFDM system which involves generating a large set of data vectors that represent the same information, where the data vector with the lowest PAPR is used for the transmission. In some SLM methods, additional information about which data is used should be transmitted to the receiver end. This will cause potential problems with decoding the signal in the presence of noise, and will obviously result in a loss in the transmission rate. We present a method to apply the SLM technique to further reduce the PAPR in the constellation developed earlier, in which the need for the transmission of side information is removed.

Assume that the data rate to be transmitted is $r$ bits per block of length- $N$ FFT symbol. Let $r_{s}$ denote the number of redundant bits of $r$ bits specifi ed for SLM $\left(r_{s} \ll r\right.$ and $r=\log _{2}(\operatorname{det}($ constellation size $\left.))\right)$. Consequently, $N_{s}=2^{r_{s}}$ constellation points should represent the same information. In this method, the input integers $I$ are mapped to the Hadamard constellation points, and the output integers are classifi ed by the sets with the same $r_{s}$ Most Signifi cant Bits (MSBs). All the corresponding constellation points in each 
set represent the same information. The IFFT operation for all these constellation points in each set is computed, and the constellation point with the lowest PAPR is transmitted.

The operation of our scheme can be described as follows. In the fi rst step, a binary information sequence is divided into blocks of $r-r_{s}$ bits. $r_{s}$ bits of zeros are added to each information block, and then it is divided into subblocks of lengths equal to $\left\{\log _{2} \sigma_{i}\right\}$ bits (refer to Fig. 3). The binary representations of these subblocks form the vector $\boldsymbol{\lambda}$ in (10). The other multiples of this vector are obtained by changing all the possible values for $r_{s}$ MSBs of the binary information sequence. Then, $N_{s}$ different Hadamard constellation points are produced by (10). The corresponding time domain OFDM signals result in various values for the PAPR. Finally, the constellation point with the lowest PAPR is selected for transmission.

All the different constellation points that represent the same information have the same $r-r_{s}$ bits. Thus, at the receiver end, the constellation point is decoded by (12), and the $r_{s}$ extra bits are discarded, since the transmitted information is in the remaining $r-r_{s}$ bits. Therefore, this method can be expressed as a variant of SLM in which no side information on the choice of the transmit signal needs to be transmitted. The degradation in the data rate can be ignored, since by using only one or two redundant bits, which are fewer than those of data rate, a signifi cant PAPR reduction is obtained.

The Hadamard constellation has a zero shaping gain ${ }^{3}$, due to its cubic boundary. $\mathrm{Nu}-$ merical results show applying the SLM method to the resulting cubic constellation and selecting the point with the lowest PAPR result in a reduction in the average energy, reflected in a small, however positive shaping gain. This justifi es our earlier claim that the reduction in the PAPR is achieved at no extra cost in terms of a reduction in the spectral effi ciency and/or an increase in the average energy of the constellation.

\footnotetext{
${ }^{3}$ Shaping gain is defi ned as the relative reduction in the required average energy for a given number of constellation points with respect to a cubic constellation.
} 


\section{Simulation Results}

In this section, we present simulations for a complex baseband OFDM system with $N=128$ subchannels employing 16-QAM by using $10^{7}$ randomly generated OFDM symbols. First, we show the PAPR performance of the Hadamard constellation. The next step is then to show the capability of the SLM technique, when it is applied to the Hadamard constellation to achieve a signifi cant PAPR reduction. Our simulation results are presented as the Complementary Cumulative Density Function (CCDF) of the PAPR of the OFDM signals. This is expressed as follows:

$$
\operatorname{CCDF}\{\operatorname{PAPR}(\mathbf{y})\}=P\{\operatorname{PAPR}(\mathbf{y})>\gamma\}
$$

This equation can be interpreted as the probability that the PAPR of a symbol block exceeds some clip level $\gamma$ (it is referred as symbol clip probability [15]).

As mentioned in Section I, the PAPR is a major problem for the time domain signal, that is, the effect of a large PAPR is in the continuous signal rather than in the discrete signal. According to (5) and (6), the continuous PAPR can be estimated by the IFFT of length $L N$ for the zero padded sequence of length $L N$. Results for the oversampling to $L=1,2$, and 4 are reflected in Fig. 4. The continuous PAPR can be approximated by the oversampling $L=4$. As mentioned in [1-3], further oversampling will result in minor improvements only. We have a PAPR reduction of more than $4 \mathrm{~dB}$ close to the $10^{-5}$ symbol clip probability.

Increasing the OFDM block length $N$ causes the PAPR of the conventional OFDM signal to increase. As plotted in Fig. 5 for the different values of $N=32,64$, and 128 , the PAPR of the OFDM system changes very little. The effect of the constellation size, when the Hadamard constellation is used in the OFDM system is also investigated. Like conventional OFDM systems, employing different constellations in each subchannel does not affect the PAPR of the OFDM signal, since all the constellation points have the same root constellation. Employing different constellations in subchannels is equivalent to having various data rates. As mentioned before, all the constellations with the lower number of points are selected from a root constellation that is defi ned by the Hadamard matrix. 
This result is exhibited in Fig. 6. Employing other modulation schemes in the previous simulations results in minor changes only of all the depicted PAPR statistics. According to these simulations, the use of the Hadamard constellation in OFDM systems as a constellation shaping method considerably reduces the PAPR with a low complexity encoding and decoding algorithm which can be easily implemented.

Fig. 7 offers the simulation results of implementing our SLM technique, applied to the Hadamard constellation in the simulated OFDM system. The PAPR probability for $r_{s}=1,2$, and 4 redundant bits is depicted.

As it is illustrated in Fig. 7, using only one bit in $4 \times 128$ bits per block of length 128 FFT symbol ${ }^{4}$ results in a $5.6 \mathrm{~dB}$ improvement in the PAPR reduction; more redundant bits further reduces the PAPR.

\section{A. Some Insight to the Achieved Performance}

In a conventional OFDM system with $N$ different subcarriers, the time domain samples can be approximated by zero mean Gaussian random variables, based on adopting the central limit theorem. Therefore, the amplitude of these samples has a Rayleigh distribution, and the CCDF of the PAPR of the OFDM signal can be approximated as follows:

$$
P\{\operatorname{PAPR}(\mathbf{y})>\gamma\}=1-\left(1-e^{-\gamma}\right)^{N}
$$

The use of $N_{s}$ statistically independent vectors that have the same information for transmission in the SLM method changes the CCDF of the PAPR of the OFDM signal such that

$$
P\{\operatorname{PAPR}(\mathbf{y})>\gamma\}=\left(1-\left(1-e^{-\gamma}\right)^{N}\right)^{N_{s}} .
$$

Therefore, in the logarithmic CCDF vs. PAPR graph, the slope of the depicted line is proportional to $N_{s}$ (see Fig. 8). By increasing the number of vectors with the same information, the slope of the CCDF vs. PAPR graph increases. Thus, the major PAPR reduction

${ }^{4} \mathrm{By}$ using 16 -QAM in a 128 channel OFDM system, there are $16^{128}=2^{4 \times 128}$ constellation points. 
is gained by the fi rst few redundant bits, and the PAPR reduction which is gained by the successively doubling of $N_{s}$ is lower in each step, as it is shown in Fig. $8\left(\Delta_{1}>\Delta_{2}>\cdots\right)$. This is the reason that we have applied the SLM technique to the Hadamard constellation. As mentioned in Section IV, the method employing only the Hadamard constellation considerably reduces the PAPR. By Adopting the Hadamard constellation in the proposed SLM method, not only can we lower the PAPR considerably, but also we can approximately maintain the slope of the CCDF vs. PAPR curve. Therefore, by using just one or two redundant bits, we can further reduce the PAPR. By using eight redundant bits in the general SLM methods in an OFDM system, we attain the same PAPR reduction as that we have achieved by using only one redundant bit in the proposed SLM method applied to the Hadamard constellation.

\section{B. Comparison}

To complete our simulations, we compare our results with some of those in recent works. In [12], an SLM method based on multiplying the constellation point by $U$ different and pseudo-random but fi xed vectors is introduced. For the same system as ours, with $N_{s}=4$ different vectors, the PAPR reduction of $3 \mathrm{~dB}$ close to $10^{-5}$ symbol clip probability is gained; however, at the same symbol clip rate, we have a $4 \mathrm{~dB}$ PAPR reduction just by using the Hadamard constellation and 6dB by using our SLM method. Also, the complexity of our algorithm is comparable with the method in [12]. The main complexity is in the encoding procedure, but due to the special recursive structure of the Hadamard matrix, the encoding algorithm can be implemented easily. Also, note that in [12] some side information needs to be sent, and receiving accurate side information is important.

In [12], a PTS method is introduced. The PAPR reduction of this method is also less than that in our method. It is possible to apply a PTS method to our constellation, especially since the PTS is considerably better with respect to PAPR reduction vs. additional system complexity (the number of IFFTs) [11,12].

The tone reservation, a well known method for PAPR reduction in multicarrier systems [34], is an effi cient PAPR reduction technique, provided it can converge quickly to 
a good PAPR solution. In [32], an effi cient approximation for the active-set approach is developed, and an excellent cost vs. performance tradeoff is obtained by using the octagonal boundary. The method in [32], compared to the work in [34], has almost the same performance in the PAPR reduction, and a faster convergence. The complexity of [32] is comparable with ours; however, we have about 3dB lower PAPR than that in [34] or [32] for the similar system parameters. Note that in the tone reservation method, some tones are reserved for the PAPR reduction and some of the tones are not used for transmitting, implying a loss in data rate.

In [35], another approach, similar to [12], is introduced for the SLM. The authors have introduced this method for MIMO-OFDM systems. The simulation results in [35] is similar to [12].

In [36], by extending the SLM method, a good PAPR reduction is achieved. A set of distinct sequences are generated from the data by using a modifi ed repeat accumulate code. For a 128 channel OFDM system, employing QPSK modulation, a 2.75dB PAPR reduction close to $10^{-3}$ symbol clip rate is gained by using a three stage Linear Feedback Shift Register (LFSR). By defi ning a clipping ratio of two an additional $2 \mathrm{~dB}$ reduction in the PAPR is achieved. However, our proposed methods based on the Hadamard constellation can lower the PAPR more (about $4 \mathrm{~dB}$ by using only the hadamard constellation, and about $5 \mathrm{~dB}$ or $6 \mathrm{~dB}$ by using one or two redundant bits in the SLM method). Note that the method introduced in [36], similar to our proposed method, can recover the data in the receiver without prior knowledge of the selected sequence.

\section{CONCLUSION}

We have proposed a constellation shaping method that achieves a substantial reduction in the PAPR in an OFDM system with a low complexity. An SLM technique is applied to this constellation to further reduce the PAPR of the OFDM signal. The proposed scheme signifi cantly outperforms other PAPR reduction techniques reported in the literature. This technique offers a PAPR about $2 \mathrm{~dB}$ to $3 \mathrm{~dB}$ lower than that of some recent works, without 
the additional costs in energy and/or spectral effi ciency. Moreover, it has a small computational complexity.

\section{REFERENCES}

[1] C. Tellambura, "Computation of the Continuous-Time PAR of an OFDM Signal with BPSK Subcarriers," IEEE Communications Letters, vol. 5, pp. 185-187, May 2001.

[2] H. Yu and G. Wei, "Computation of the continuous-time PAR of an OFDM signal," in International Conference on Acoustics, Speech and Signal Processing (ICASSP'03), Hong Kong, China, 6-10 April 2003, IEEE, pp. IV-529-31, IEEE.

[3] H. Ochiai and H. Imai, "Performance Analysis of Deliberately Clipped OFDM Signals," IEEE Trans. on Commun., vol. 50, pp. 89-101, Jan. 2002.

[4] J. A. Davis and J. Jedwab, "Peak-to-mean power control in OFDM, Golay complementary sequences, and ReedMuller codes," IEEE Trans. Inform. Theory, vol. 45, no. 7, pp. 2397-2417, Nov. 1999.

[5] K. Patterson, "Generalized Reed-Muller codes and power control in OFDM modulation," IEEE Trans. Inform. Theory, vol. 46, no. 1, pp. 104-120, Jan. 2000.

[6] H. Saeedi, M. Sharif, and F. Marvasti, "Clipping noise cancellation in OFDM systems using oversampled signal reconstruction,” IEEE Comm. Lett., vol. 6, pp. 73-75, Feb. 2002.

[7] D. Wulich and L. Goldfeld, "Reduction of peak factor in orthogonal multicarrier modulation by amplitude limiting and coding," IEEE Trans. on Commun., vol. 47, no. 1, pp. 18-21, Jan. 1999.

[8] X. Li and L. J. Cimini, "Effects of clipping and fi ltering on the performance of OFDM," IEEE Comm. Lett., vol. 2, no. 5, pp. 131-133, May 1998.

[9] M. Sharif, M. Gharavi-Alkhansari, and B. H. Khalaj, "On the peak-to-average power of OFDM signals based on oversampling," IEEE Trans. on Comm., vol. 51, pp. 72-78, Jan. 2003.

[10] H. Ochiai and H. Imai, “On the Distribution of the Peak-to-Average Power Ratio in OFDM Signals," IEEE Trans. on Commun., vol. 49, no. 2, pp. 282-289, Feb. 2001.

[11] S. H. Muller and J. B. Hubber, "OFDM with reduced peak-to-average power ratio by optimum combination of partial transmit sequences," Electron. Lett., vol. 33, no. 5, pp. 368-369, Feb. 1997.

[12] M. Breiling, S. H. Muller-Weinfurtner, and J. B. Hubber, "SLM Peak-Power Reduction Without Explicit Side Information,” IEEE Commun. Lett., vol. 5, no. 6, pp. 239-241, June 2001. 
[13] K. Yang and S. Chang, "Peak-to-Average Power Control in OFDM Using Standard Arrays of Linear Block Codes," IEEE Commun. Lett., vol. 7, no. 4, pp. 174-176, Apr. 2003.

[14] N. Carson and T. A. Gulliver, "Peak-to-Average Power Ratio Reduction of OFDM Using Repeat-Accumulate Codes and Selective Mapping,” 2002 IEEE International Symposium on Information Theory (ISIT 2002), p. 244, June 30-July 52002.

[15] B. S. Krongold and D. L. Jones, "PAR Reduction in OFDM via Active Constellation Extension," IEEE Trans. Broadcasting, vol. 49, no. 3, pp. 258-268, Sept. 2003.

[16] H. K. Kwok, Shape Up: Peak-Power Reduction via Constellation Shaping, Ph.D. thesis, University of Illinois, 2001.

[17] H. K. Kwok and D. L. Jones, "PAR Reduction for Hadamard Transform-Based OFDM," in 34th Conference on Signal, Systems, and Computers, Princeton, NJ, March 15-17 2000.

[18] H. K. Kwok and D. L. Jones, "PAR Reduction via Constellation Shaping," in 2000 International Symposium on Information Theory, Sorrento, Italy, June 25-30 2000.

[19] H. Cohen, Graduate Texts in Mathematics: A Course in Computational Algebraic Number Theory, vol. 138, SpringVerlag, 1993.

[20] H. J. S. Smith, “On systems of linear indeterminate equations and congruences," Phil. Trans. Roy. Soc. London, vol. 151, pp. 293-326, 1861.

[21] M. Newman, “The Smith Normal Form,” Linear ALgebra Appl., vol. 254, pp. 367-381, 1997.

[22] Arne Storjohann and George Labahn, "A Fast Las Vegas Algorithm for Computing the Smith Normal Form of a Polynomial Matrix,” Technical Report CS-94-43, University of Waterloo, Nov. 1994.

[23] R. Bernardini and R. Manduchi, "On the Reduction of Multidimensional DFT to Separabile DFT by Smith Normal form Theorem," Signal Processing Letter, vol. 5, no. 3, May-June 1994.

[24] Arne Storjohann, Computation of Hermite and Smith Normal Forms of Matrices, Ph.D. thesis, University of Waterloo, 1994.

[25] R. Kannan and A. Bachem, "Polynomial algorithms for computing the Smith and Hermit normal forms of an integer matrix,” SIAM J. Comp., vol. 8, pp. 499-507, 1981.

[26] R. Kannan, "Polynomial-time algorithms for solving systems of linear equations over polynomials," Theoretical Comp. Sci., vol. 39, pp. 69-88, 1985.

[27] E. Kaltofen and B. D. Saunders, "Parallel algorithms for matrix normal forms," Linear Algebra and Its Applications 
136, pp. 189-208, 1990.

[28] P. Y. Cochet and R. Serpollet, "Digital transformfor a selective channel estimation," in the IEEE International Conference on Communication. IEEE, 1998, IEEE.

[29] R. W. Bauml, R. F. H. Fischer, and J. B. Huber, "Reducing the peak-to-average power ratio of multicarrier modulation by selected mapping," Electronic Letters, vol. 32, pp. 2056-2057, 1996.

[30] J. V. Eatvelt, G. Wade, and M. Tomlinson, "Peak to average power reduction for OFDM schemes by selective scrambling,” Electronic Letters, vol. 32, pp. 1963-1964, 1996.

[31] H. Nikopour, A. K. Khandani, and S. H. Jamali, "Turbo Coded OFDM Transmission over Nonlinear Channel," Submitted to IEEE Trans. on Inform. Theory, 2004.

[32] B. S. Krongold and D. L. Jones, “An Active-Set Approach for OFDM PAR Reduction via Tone Reservation," IEEE Trans. on Signal Processing, vol. 52, no. 2, pp. 495-509, Feb. 2004.

[33] G. D. Forney and M. D. Trott, "The dynamics of group codes: state spaces, trellis diagrams, and canonical encoders,” IEEE Trans. Inform. Theory, vol. IT-39, pp. 1491-1513, Sept. 1993.

[34] J. Tellado, Peak to average power reduction for multicarrier modulation, Ph.D. thesis, Stanford University, Stanford CA, 2000.

[35] Yung-Lyul Lee, Young-Hwan You, Won-Gi Jeon, Jong-Ho Paik, and Hyoung-Kyu Song, "Peak-to-Average Power Ratio in MIMO-OFDM Systems Using Selective Mapping,” IEEE Comm. Lett., vol. 7, no. 12, pp. 575-577, Dec. 2003.

[36] N. Carson and T. A. Gulliver, "Performance of OFDM With Modifi ed RA Codes and Clipping," IEEE Comm. Lett., vol. 7, no. 9, pp. 440-442, Sep. 2003. 


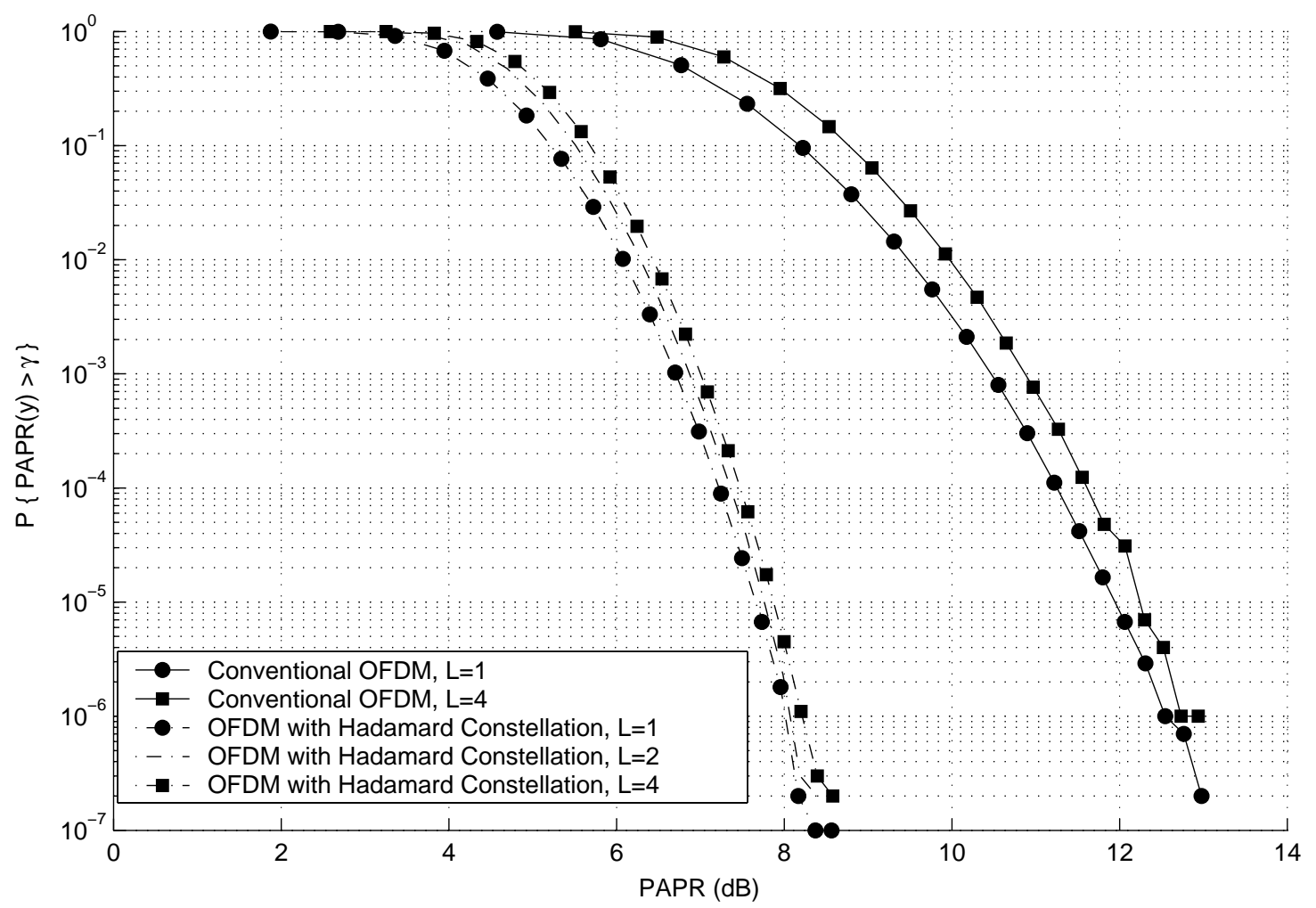

Fig. 4. CCDF of PAPR by using Hadamard constellation in a 128 channel OFDM system employing 16-QAM constellation with different oversampling factors for continuous PAPR approximation. 


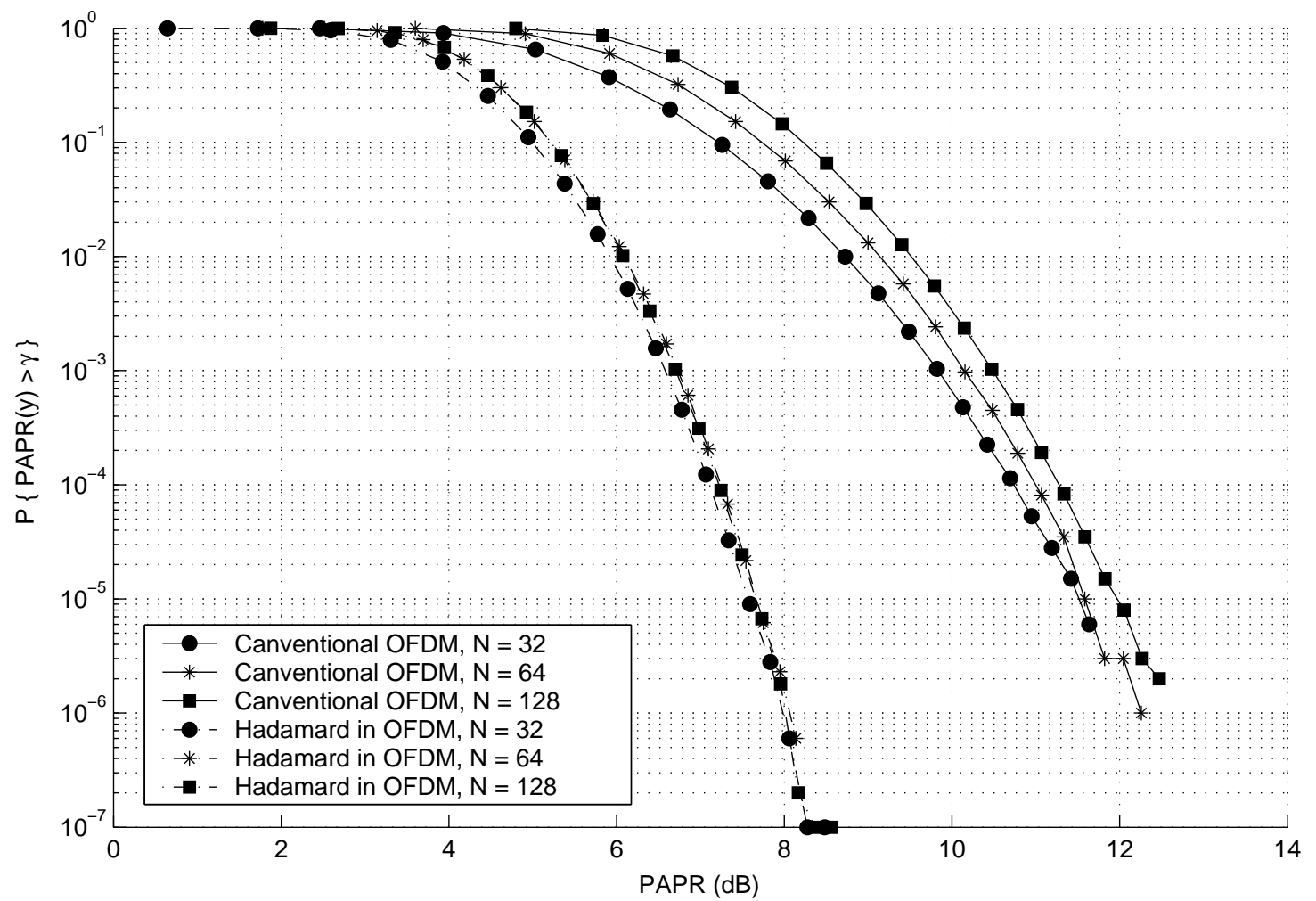

Fig. 5. CCDF of PAPR by using Hadamard constellation in an $N$ channel OFDM system employing 16-QAM constellation. 


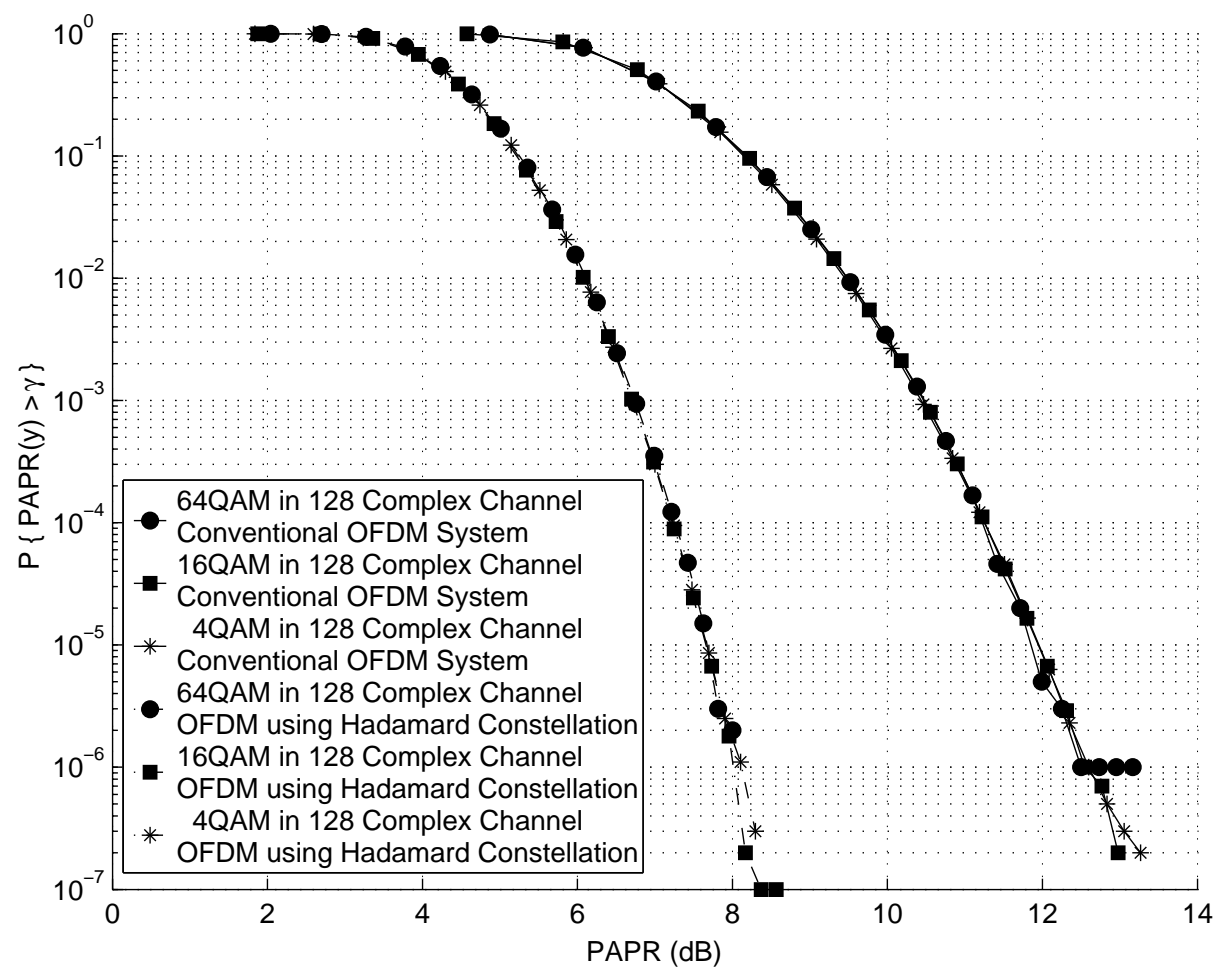

Fig. 6. CCDF of PAPR by using Hadamard constellation in a 128 channel OFDM system employing different QAM constellations. 


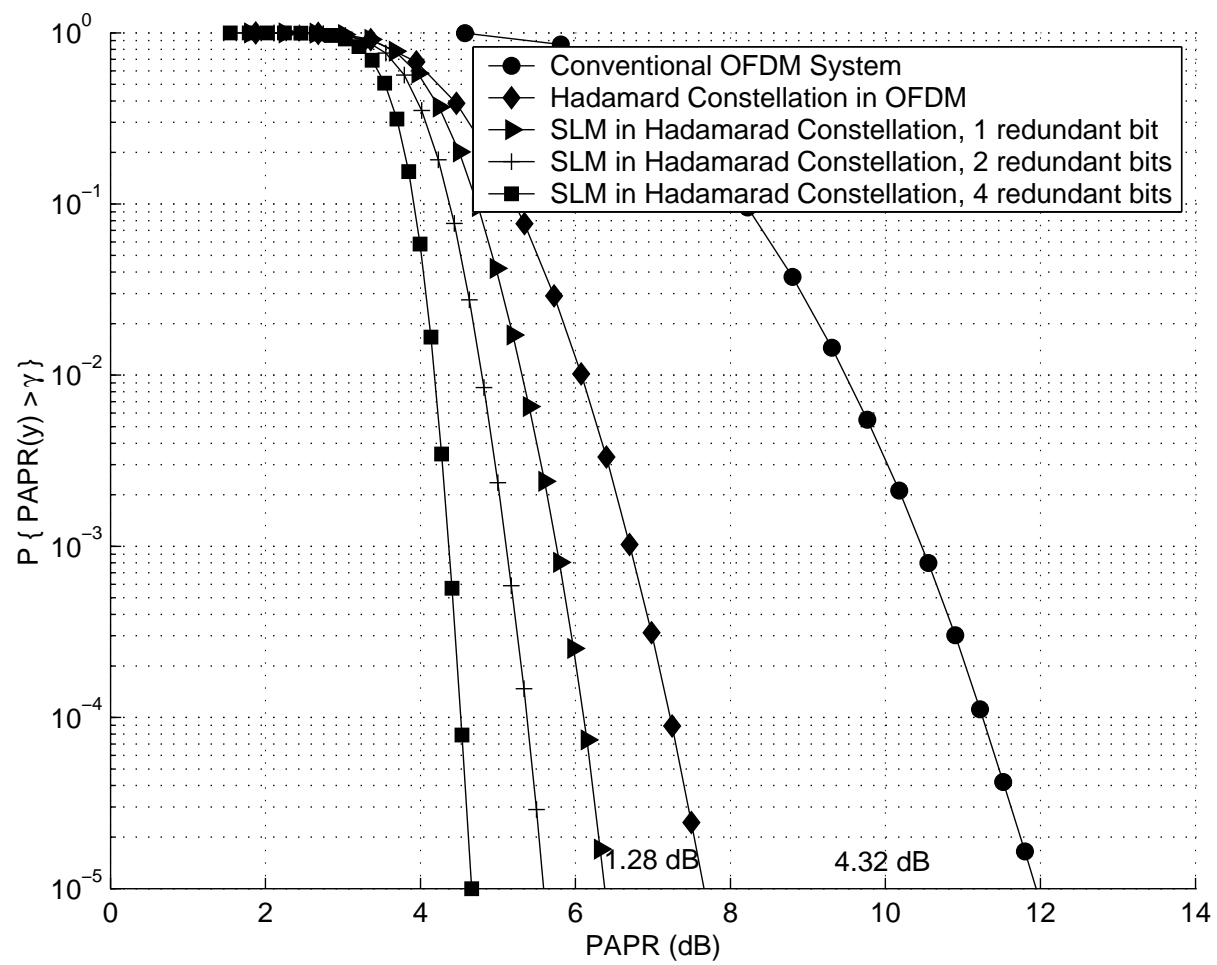

Fig. 7. CCDF of PAPR by SLM method based on Hadamard constellation in a 128 channel OFDM system employing 16-QAM constellation. 


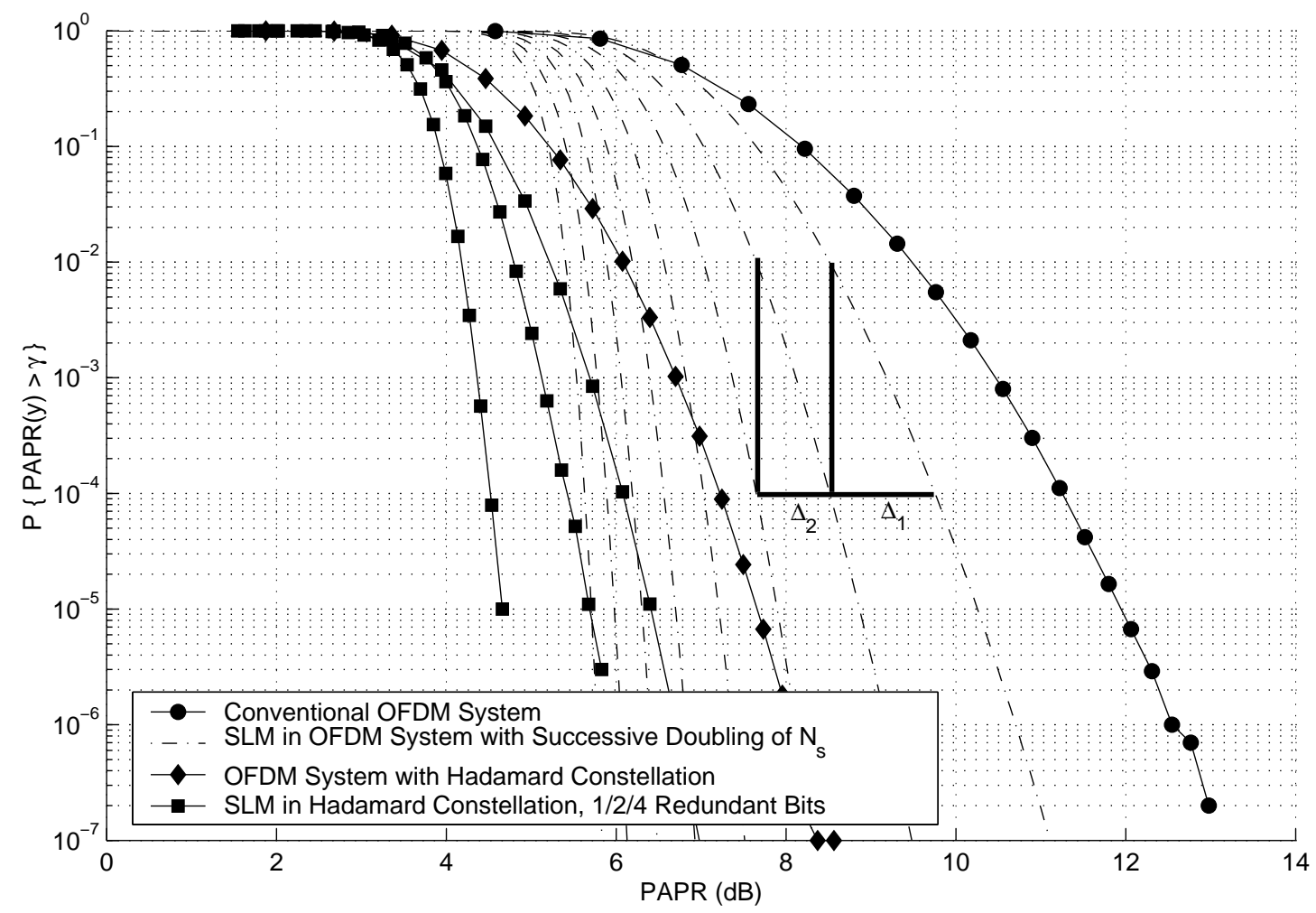

Fig. 8. CCDF of PAPR in a 128 channel OFDM system with SLM method using different number of redundant bits. 